\title{
Determinação da densidade corporal por equações generalizadas: facilidade e simplificação no método
}

\section{Determination of body density by generalized equations: easiness and simplification in the method}

\author{
Andrey Jorge Serral; Alessandra Marcolongo do Amaral'; Roberta Lukcevicius Rica ${ }^{3}$; Nilva \\ Petrina Barbieri²; Dermeval Reis Junior ${ }^{5}$; José Antonio Silva Junior ${ }^{4}$; Danilo Sales Bocalini ${ }^{6}$ \\ ${ }^{1}$ Especialista em Fisiologia do Exercício; Doutorando em Cardiologia - UNIFESP. \\ ${ }^{2}$ Professor de Ensino Fundamental e Médio - Secretaria de Educação do Estado de São Paulo. \\ ${ }^{3}$ Especialista em Personal Training - UniFMU \\ Doutor em Ciências Biológicas-UNIFESP. \\ ${ }^{5}$ Especialista em Fisiologia Humana e Mestrando em Ciências Fisiológicas - UNIFESP \\ Mestre e Doutorando em Ciências Fisiológicas - UNIFESP. \\ Endereço para correspondência \\ Andrey Jorge Serra \\ R. Manoel José Viana, 01 \\ 03731-124 - SP [Brasil] \\ andrey@fcr.epm.br
}

\begin{abstract}
Resumo
A determinação da densidade corporal (DC) por dobras cutâneas é amplamente utilizada em todo mundo. Examinou-se a concordância entre o somatório de sete (SSDC) e três (STDC) dobras cutâneas para determinação da DC. A DC foi avaliada em 72 homens e 93 mulheres, com idade de 18 a 58 anos. Avaliouse a concordância entre as mensurações com teste " $t$ " pareado, correlação de Pearson e Bland-Altman. A DC foi similar para homens (SSDC: 1,043 $\pm 0,014$; STDC: $1,043 \pm 0,015$ ) e mulheres (SSDC: $1,025 \pm 0,014$; STDC: $1,026 \pm 0,016$ ), com uma correlação excelente (homens: 0,97; mulheres: 0,92). Bland-Altman indicou valores ótimos para homens (Bias: 0,0007 $\pm 0,0035)$ e mulheres (Bias: 0,0004 $\pm 0,0064$ ), com discordância mínima (homens: -0,0062 a 0,0077; mulheres: $-0,0121$ a 0,0130 ). Conclui-se que o uso de apenas três dobras cutâneas é aceitável para determinação da DC, com a vantagem de otimização do tempo despendido para avaliação.
\end{abstract}

Descritores: Composição corporal; Densidade corporal; Equações generalizadas; Percentual de gordura corporal.

\begin{abstract}
The determination of body density (BD) by skinfold thickness measurements is broadly used around the world. It was examined the agreement between two equations that use seven (SS) and three (TS) skinfold thickness measurements to assess the BD. The BD was evaluated in 72 men and 93 women aged 18-58 years. Paired Student t-test, Pearson's correlation, and Bland-Altman analysis were used to evaluate agreement between the measurements. The BD was highly similar to men (SS: $1.043 \pm 0.014$; TS: $1.043 \pm 0.015)$ and women (SS: 1.025 \pm 0.014 ; TS: $1.026 \pm 0.016$ ), with excellent correlations (men: 0.97; women: 0.92). The Bland-Altman analysis indicated excellent agreement for men (bias: 0.0007 $\pm 0.0035)$ and women (bias: $0.0004 \pm 0.0064)$, with minimal disagreement (men: -0.0062 to 0.0077 ; women: -0.0121 to 0.0130 ). This study showed that the use of only three skinfold measurements is acceptable for determination of $\mathrm{BD}$, with the advantage of optimization of the time spent for evaluation.
\end{abstract}

Key words: Body composition; Body density; Body fat percentage; Generalized equations. 


\section{Introdução}

A análise da densidade corporal (DC) é uma ferramenta essencial para quantificar e monitorar alterações em diferentes compartimentos corporais, incluindo músculo esquelético, ossos, gordura e água. Nesse sentido, a divisão corporal em dois compartimentos distintos, peso corporal de gordura e livre de gordura, tem sido amplamente pragmatizada ${ }^{1}$. Na impossibilidade de mensuração direta da $\mathrm{DC}$, diversos métodos indiretos foram propostos para tal $\mathrm{fim}^{2}$. Um método bem difundido para predizer a DC é a espessura de dobras cutâneas que, por sua simplicidade de aplicação, custo baixo e relativa precisão, resultou no desenvolvimento de mais de cem equações documentadas na literatura ${ }^{3}$.

As equações propostas por Jackson, Pollock e Ward ${ }^{4,5}$ para predizer a DC em homens e mulheres são amplamente populares entre profissionais envolvidos com avaliação física. Tais equações predizem, com acurácia, a DC em pessoas com diferentes idades e quantidade de gordura corporal, como foi constatado por-meio de expressivos índices de correlação para homens $(r>0,90)$ e mulheres $(r>0,81)$ avaliados em pesagem hidrostática. Além disso, em estudo conduzido no Brasil, um erro predito de apenas 3,29\% foi encontrado na determinação do percentual de gordura corporal (baseada na DC), quando comparado com avaliações realizadas por medida de absorção dos raios $X$ de dupla energia ${ }^{6}$.

Jackson, Pollock e Ward ${ }^{4,5}$ padronizaram equações capazes de estimar a DC utilizando a soma de sete, quatro e três dobras cutâneas. A correlação entre as diferentes somas foi alta $(r>0,83)$, demonstrando que a utilização de três dobras cutâneas já oferece valores confiáveis sobre a DC. Contudo, o uso de apenas três dessas dobras, para tal determinação, deve ser avaliado em diferentes populações, procedimento sustentado por argumentos que consideram a validade de equações em que se utiliza esse método, restrita para a população da qual essas equações foram derivadas ${ }^{7}$. Neste estudo, examinou-se a concordância entre duas equações generaliza- das, envolvendo o somatório de sete (SSDC) e três (STDC) dobras cutâneas, para determinação da DC na população brasileira.

\section{Materiais e métodos}

\section{Realização da pesquisa e casuística}

Neste estudo observacional-retrospectivo, analisaram-se 165 prontuários de avaliações físicas de indivíduos da academia de ginástica "Fit. One", localizada na cidade de São Paulo - SP, no bairro Vila Mariana. A amostra contemplou homens e mulheres com diferentes características de composição corporal, idade e nível de atividade física (Tabela 1). O estudo seguiu as normas éticas exigidas pela Resolução no 196/1996 do Conselho Nacional de Saúde e foi aprovado pelo Comitê de Ética em Pesquisa da Unifesp (Parecer 1847/08).

\section{Avaliação antropométrica}

A massa corporal (MC) foi avaliada em balança mecânica da marca Filizola (modelo 31), com capacidade de até $150 \mathrm{~kg}$ e sensibilidade de 100 g. No momento da avaliação, os indivíduos estavam descalços e vestiam o mínimo de roupas. A estatura (ES) foi mensurada em estadiômetro SANNY (modelo ES-2030), fixado à parede e calibrado em escala de $1 \mathrm{~mm}$. Considerando-se a razão entre $\mathrm{MC}$ e o quadrado da medida da ES, o IMC foi derivado:

$$
\mathrm{IMC}=\mathrm{MC} \div \mathrm{ES}^{2}
$$

A espessura das dobras cutâneas foi mensurada nas regiões subescapular, suprailíaca, axilar-média, peitoral, tricipital, abdominal e coxa, com um compasso específico Lange. Todas as medidas foram realizadas no segmento corporal direito e atenderam às recomendações previamente estabelecidas ${ }^{4,5}$. Consequentemente, 
Tabela 1: Características descritivas da amostra

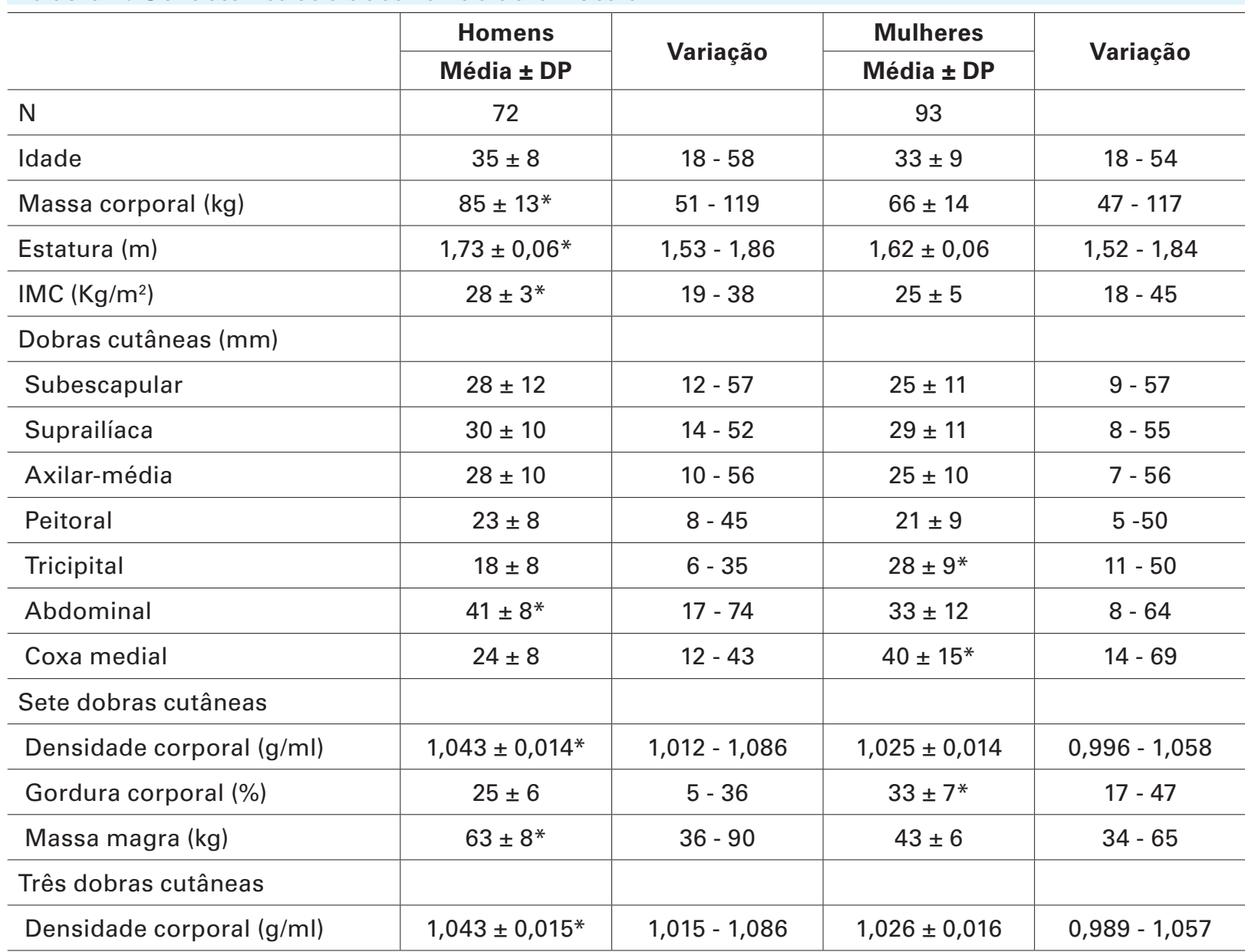

Teste "t" pareado foi aplicado para comparação entre os valores médios dos diferentes constituintes corporais: DC, percentual de gordura e massa magra preditos conforme os protocolos de sete e três dobras cutâneas $(\mathrm{P}>0.05$, para todas as comparações). Somente foram encontradas diferenças significantes na comparação entre os gêneros (Teste " $t$ " não pareado; * $\mathrm{P}<0,001)$

foi possível calcular a DC, empregando-se as seguintes equações:

a) somatório de sete dobras cutâneas (SSDC);

Homens: $\mathrm{DC}=1,11200000-0,00043499\left(\mathrm{X}_{1}\right)$ $+0,00000055\left(X_{1}\right)^{2}-0,00028826\left(X_{2}\right)$;

Mulheres: $\mathrm{DC}=1,0970-0,00046971\left(\mathrm{X}_{1}\right)+$ $0,00000056\left(X_{1}\right)^{2}-0,00012828\left(X_{2}\right)$;

b) somatório de três dobras cutâneas (STDC);

Homens: $\mathrm{DC}=1,1093800-0,0008267\left(\mathrm{X}_{3}\right)+$ $0,0000016\left(X_{3}\right)^{2}-0,0002574\left(X_{2}\right)$;

Mulheres: $\mathrm{DC}=1,0994921-0,0009929\left(\mathrm{X}_{4}\right)+$ $0,0000023\left(X_{4}\right)^{2}-0,0001395\left(X_{2}\right)$;

$X_{1}=$ soma das sete dobras cutâneas, $X_{2}=$ idade; $X_{3}=$ soma das dobras cutâneas da coxa, peitoral e abdominal; $\mathrm{X}_{4}$ : soma das dobras cutâneas do tricipital, coxa e suprailíaca.

Derivada a DC, o percentual de gordura corporal (\%G) foi calculado mediante equação proposta por Siri (1956): \%G $=(495 \div \mathrm{DC})-450$

\section{Anólise estatística}

Avaliaram-se os dados com pacote computadorizado GraphPad Prism 4.0. A concordância entre os valores de DC, estimados conforme a SSDC e a STDC, foi analisada de acordo com os seguintes procedimentos: (a) teste " $\mathrm{t}$ " Student para dados pareados; (b) co- 
eficientes de correlação de Pearson; (c) dispersão por Bland-Altman. Teste " $\mathrm{t}$ " não pareado foi aplicado na comparação de diferentes varáveis entre os gêneros. Todos os resultados são expressos como média \pm desvio-padrão, com nível de $\mathrm{P}<0,05$.

\section{Resultados}

A Tabela 1 apresenta as características individuais, com as respectivas variáveis analisadas. Não houve diferenças significantes nos valores médios para idade entre homens e mulheres. Todavia, os valores médios referentes à MC, à ES e ao IMC foram significativamente mais elevados nos indivíduos do gênero masculino. Ao considerar as medidas de dobras cutâneas, os resultados apontaram valores médios de espessura, estatisticamente superiores, nas regiões tricipital e coxa, entre as mulheres, e somente na região abdominal, entre os voluntários masculinos.

As análises da composição corporal revelaram que os valores de DC e massa magra, em homens, foram significativamente superiores quando comparados aos valores encontrados em mulheres, diferença que mostrou ser independente da equação de predição utilizada (Tabela 1). Em contrapartida, as mulheres apresentaram valores médios de \%G mais elevados que os homens, como estimados em cada uma das equações. Além disso, numa análise pareada com teste " $\mathrm{t}$ " Student, a comparação entre SSDC e STDC mostrou que ambos os protocolos identificam valores similares de DC, \%G e massa magra em ambos os sexos (Tabela 1).

Na Figura 1 estão dispostos os coeficientes de correlação de Pearson para DC estimada conforme os protocolos de SSDC e STDC. Os resultados demonstram que as duas equações identificam valores muito similares de DC, alcançando expressivos índices de correlação, com coeficientes de 0,97 para homens, e de 0,92 para mulheres.
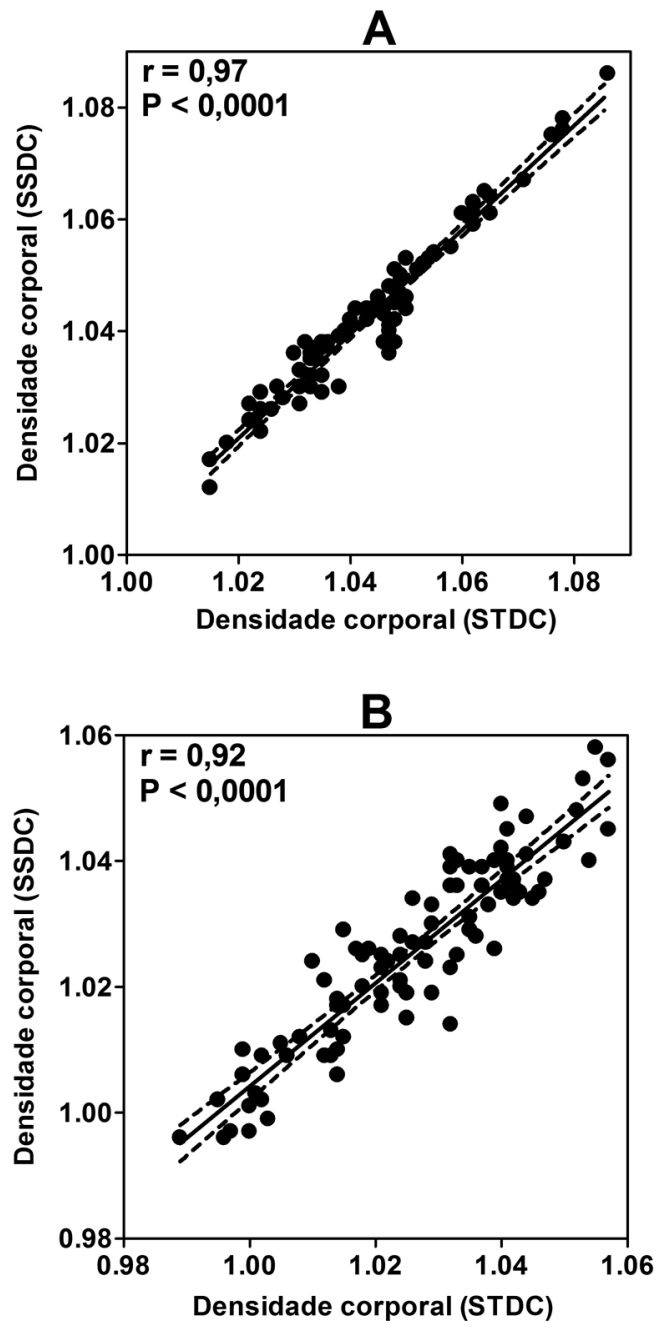

Figura 1: Correlação entre as equações de somatório de sete (SSDC) e três (STDC) dobras cutâneas para determinação da DC. Nos painéis $\mathrm{A}$ e $\mathrm{B}$ estão dispostas as correlações para homens e mulheres, respectivamente. O valor de correlação (r) e o nível de significôncia também são mostrados. As linhas contínuas representam as regressões lineares e as linhas pontilhas mostram os intervalos de confiança de $95 \%$

Posteriormente, a concordância entre as medidas foi avaliada por plotagem gráfica com Bland-Altman (Figura 2). As análises mostraram concordância satisfatória entre SSDC e STDC para homens (bias: 0,00077 $\pm 0,0035$; limite de concordância de $95 \%$ : $-0,0062$ a 0,0077) e para mulheres (bias: 0,0004 \pm 0,0064; limite de concordância de 95\%: -0,0121 a 0,0130). 

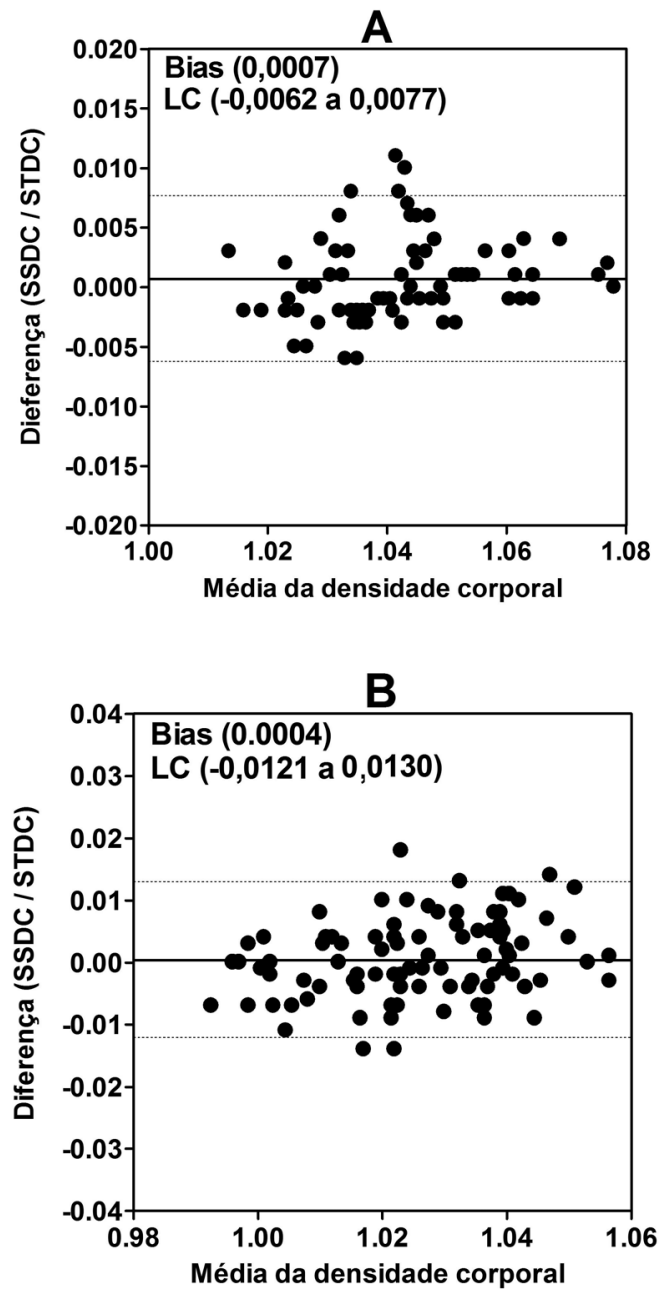

Figura 2: Grófico de Bland-Altman para verificar o grau de concordôncia entre somatória de sete (SSDC) e três (STDC) dobras cutâneas quanto à densidade corporal. Nos painéis A e B estão dispostas as dispersões para homens e mulheres, respectivamente. $O$ valor médio do bias e os limites de concordância de $95 \%$ (LC) também são mostrados. As linhas contínuas representam o bias e as linhas pontilhas mostram os LC

\section{Discussão}

O uso de dobras cutâneas é um método bem difundido para avaliação da DC, em especial para determinação do teor de gordura corporal $^{2,7,9}$. O custo baixo, inocuidade e simplicidade de aplicação em grandes populações são fatores que contribuem para que essa me- todologia possa ser identificada em centenas de trabalhos publicados na literatura ${ }^{2,3}$.

Há diversas equações disponíveis para estimar a DC, mas as propostas por Jackson, Pollock e Ward ${ }^{4,5}$ estão entre as mais difundidas. Essas equações, conhecidas como generalizadas, foram validadas em grandes populações, com diferentes idades, nível de atividade física e composição corporal ${ }^{4,5,10}$. A DC é estimada pela mensuração de sete dobras cutâneas; no entanto, a utilização de apenas três surge como alternativa para facilitar o processo de avaliação.

Neste estudo, demonstrou-se que os valores médios de DC são similares entre as equações de SSDC e STDC, como avaliado pelo teste " $\mathrm{t}$ " Student pareado. Além disso, nossos achados são condizentes com estudos prévios ${ }^{4}$ 5 , visto que uma significativa correlação entre as duas equações foi também identificada, com valores iguais a 0,97 para homens, e 0,92 para mulheres.

Posteriormente, utilizou-se a técnica de Bland-Altman para analisar o limite de concordância entre as duas equações. Essa providência foi adotada, uma vez que o coeficiente de correlação de Pearson reflete a força de uma relação entre duas variáveis, não necessariamente a concordância entre elas ${ }^{11,12}$. Assim, os resultados consolidam que há adequada concordância entre SSDC e STDC para determinação da DC em homens e mulheres. Os diagramas de dispersão indicaram diferenças médias entre SSDC e STDC próximas de zero, com intervalos de confiança muito estreitos (Figura 2A/B).

Em suma, a análise pareada por teste " $\mathrm{t}$ " Student, a correlação de Pearson e a dispersão gráfica por Bland-Altman, aplicadas aos dados obtidos neste estudo, legitimam o uso da STDC como método aceitável para determinação da DC, com a vantagem de otimização do tempo despendido para avaliação. Além disso, em uma análise de validação cruzada, os valores derivados do uso de três dobras cutâneas mostraram ser similares a outras 17 equações ${ }^{5}$. 


\section{Conclusões}

Os resultados deste estudo sugerem a adoção da STDC para avaliação da composição corporal em homens e mulheres. Os valores de DC derivados da STDC mostraram significativos níveis de concordância quando confrontados com os provenientes da SSDC. Portanto, o uso de três dobras cutâneas parece ser uma medida interessante para estimativa da DC, principalmente quanto à economia de tempo despendido para avaliação em grandes populações.

\section{Referências}

1. McArdle WD, Katch FI, Katch VL. Fisiologia do exercício: energia, nutrição e desempenho humano. Rio de Janeiro: Guanabara Koogan; 1998.

2. Brodie DA. Techniques of measurement of body composition (Part II). Sports Med. 1988;5:74-98.

3. Jackson AS. Research design as analysis of data procedures for predicting body density. Med Sci Sports Exerc. 1984;16:616-20.

4. Jackson AS, Pollock ML. Generalized equations for predicting body density of men. Br J Nutr. 1978;40:497-504.
5. Jackson AS, Pollock ML, Ward A. Generalized equations for predicting body density of women. Med Sci Sports Exerc. 1980;12:175-81.

6. Bottaro MF, Heyward VH, Bezerra RFA, Wagner DR. Skinfold method vs dual-energy x-ray absorptiometry to assess body composition in normal and obese women. J Exerc Physiol online. 2002;5:11-8.

7. Rezende FAC, Rosado LEFPL, Priore SE, Fraceschini SCC. Aplicabilidade de equações na avaliação da composição corporal da população brasileira. Rev Nutr. 2006;19:357-67.

8. Siri WE. The gross composition of the body. Adv Biol Med Phy. 1956;4:239-80.

9. Eaton AW, Israel RG, O'Brien KF, Hortobagyi T, MacCammon MR. Comparison of four methods to assess body composition in women. Eur J Clin Nutr. 1993;47:353-60.

10. Fonseca PHS, Marins JCB, Silva AT. Validação de equações antropométricas que estimam a densidade corporal em atletas profissionais de futebol. Rev Bras Med Esporte. 2007;13:153-56.

11. Bland JM, Altman DG. Statistical methods for assessing agreement between two methods of clinical measurement. Lancet. 1986; 8:307-10.

12. Brazdzionyte J, Macas A. Bland-Altman analysis as an alternative approach for statistical evaluation of agreement between two methods for measuring hemodynamics during acute myocardial infarction. Medicina (Kaunas). 2007;43:208-14. 